\title{
LEFT ANNIHILATORS CHARACTERIZED BY GPIS
}

\author{
TSIU-KWEN LEE
}

\begin{abstract}
Let $R$ be a semiprime ring with extended centroid $C, U$ the right Utumi quotient ring of $R, S$ a subring of $U$ containing $R$ and $\rho_{1}, \rho_{2}$ two right ideals of $R$. In the paper we show that $l_{S}\left(\rho_{1}\right)=l_{S}\left(\rho_{2}\right)$ if and only if $\rho_{1}$ and $\rho_{2}$ satisfy the same generalized polynomial identities (GPIs) with coefficients in $S C$, where $l_{S}\left(\rho_{i}\right)$ denotes the left annihilator of $\rho_{i}$ in $S$. As a consequence of the result, if $\rho$ is a right ideal of $R$ such that $l_{R}(\rho)=0$, then $\rho$ and $U$ satisfy the same GPIs with coefficients in the two-sided Utumi quotient ring of $R$.
\end{abstract}

This paper is motivated by Chuang's paper [3] and Beidar's paper [2]. Recall that a ring $R$ is said to be a left faithful ring if, for $a \in R, a R=0$ implies $a=0$. For a left faithful ring $R$, the right Utumi quotient ring of $R$ can be characterized as a ring $U$ satisfying the following axioms:

(1) $R$ is a subring of $U$.

(2) For each $a \in U$, there exists a dense right ideal $\rho$ of $R$ such that $a \rho \subseteq R$.

(3) If $a \in U$ and $a \rho=0$ for some dense right ideal $\rho$ of $R$, then $a=0$.

(4) For any dense right ideal $\rho$ of $R$ and for any right $R$-module homomorphism $\phi: \rho_{R} \rightarrow R_{R}$, there exists $a \in U$ such that $\phi(x)=a x$ for all $x \in \rho$.

Let $R$ be a left faithful ring and $\rho$ be a dense right ideal of $R$. We note that $\rho$ itself is a left faithful ring. Furthermore, $\rho$ and $R$ have the same right Utumi quotient ring. More precisely, denote by $U(R)(U(\rho)$ resp.) the right Utumi quotient ring of $R$ ( $\rho$ resp.). Then there exists a ring isomorphism $h$ from $U(\rho)$ onto $U(R)$ such that $h(x)=x$ for all $x \in \rho$. In [3] Chuang proved the theorem: Let $R$ be a prime ring, $U$ its right Utumi quotient ring and $N_{R}$ a dense $R$-submodule of $U_{R}$. Then $N$ and $U$ satisfy the same generalized polynomial identities (GPIs) with coefficients in $U$. In this theorem we note that $N \cap R$ is always a dense right ideal of $R$. Since $N \cap R$ and $R$ have the same right Utumi quotient ring, Chuang's theorem just says that $R$ and $U$ satisfy the same GPIs with coefficients in $U$. Also, in an earlier paper [2] Beidar proved that the same result remains true for semiprime rings. For a semiprime ring $R$ we observe that $N \cap R$ is a dense right ideal of $R$ for any dense $R$-submodule $N_{R}$ of $U_{R}$. Also, $l_{U}(N \cap R)$, the left annihilator of $N \cap R$

Received by the editors April 11, 1994 and, in revised form, July 8, 1994; originally communicated to the Proceedings of the AMS by Ken Goodearl.

1991 Mathematics Subject Classification. Primary 16R50.

Key words and phrases. Semiprime ring, prime ring, Utumi quotient ring, GPI, orthogonal completion. 
in $U$, is zero. In this paper we shall compare two left annihilators of two right ideals $\rho_{1}$ and $\rho_{2}$ of $R$ in $U$ by considering the GPIs satisfied by the two right ideals $\rho_{1}$ and $\rho_{2}$. From this we are able to generalize Chuang's and Beidar's results. For instance, if $R$ is a semiprime ring and $\rho$ is a right ideal of $R$ such that $l_{R}(\rho)=0$, we shall prove that $\rho$ and $U$ satisfy the same GPIs with coefficients in $Q$, the two-sided Utumi quotient ring of $R$. More explicitly, we prove in this paper the following

Main Theorem. Let $R$ be a semiprime ring with extended centroid $C, U$ its right Utumi quotient ring, $S$ a subring of $U$ containing $R$ and $\rho_{1}, \rho_{2}$ two right ideals of $R$. Then $l_{S}\left(\rho_{1}\right)=l_{S}\left(\rho_{2}\right)$ if and only if $\rho_{1}$ and $\rho_{2}$ satisfy the same GPIs with coefficients in $S C$.

Throughout the paper, rings are always associative but not necessarily with unity. We shall fix some notation. For a semiprime ring $R$ we denote by $U$ its right Utumi quotient ring, by $Q$ its two-sided Utumi quotient ring and by $C$ the extended centroid of $R . U *_{C} C\left\{X_{1}, X_{1}, \ldots\right\}$ stands for the free product of the $C$-algebra $U$ and $C\left\{X_{1}, X_{2}, \ldots\right\}$, the free $C$-algebra with indeterminates $X_{1}, X_{2}, \ldots$. For these definitions and their basic properties we refer to [3], [4] and [6]. To prove the Main Theorem we need several lemmas. We begin the proof with the following easy observations.

Lemma 1. Let $R$ be a simple Artinian ring and $\rho_{1}, \rho_{2}$ be two right ideals of $R$. Then $l_{R}\left(\rho_{1}\right)=l_{R}\left(\rho_{2}\right)$ if and only if $\rho_{1}=\rho_{2}$.

Proof. Since every right ideal of a simple Artinian ring is generated by one idempotent, there are two idempotents $e$ and $f$ in $R$ such that $\rho_{1}=e R$ and $\rho_{2}=f R$. Assume that $l_{R}\left(\rho_{1}\right)=l_{R}\left(\rho_{2}\right)$. Then $1-e \in l_{R}\left(\rho_{1}\right)$ and hence $(1-e) f R=0$. This implies that $(1-e) f=0$. That is, $f=e f$. Now, $\rho_{2}=f R=e f R \subseteq \rho_{1}$. Similarly, $\rho_{1} \subseteq \rho_{2}$. Therefore $\rho_{1}=\rho_{2}$. Of course, the converse is trivial. The proof is now complete.

Lemma 2. Let $R$ be a semiprime ring and $\rho$ be a right ideal of $R$. Then $\rho$ and $\rho U$ satisfy the same GPIs with coefficients in $U$.

Proof. Let $f\left(X_{1}, \ldots, X_{t}\right) \in U *_{C} C\left\{X_{1}, X_{2}, \ldots\right\}$ be a GPI satisfied by $\rho$. Fix $y_{1}, \ldots, y_{t} \in \rho U$. Write $y_{i}=\sum_{j=1}^{n(i)} a_{i j} u_{i j}$, where $a_{i j} \in \rho$ and $u_{i j} \in U, 1 \leq i \leq$ $t$. Since $\rho R \subseteq R, f\left(\sum_{j=1}^{n(1)} a_{1 j} Y_{1 j}, \ldots, \sum_{j=1}^{n(t)} a_{t j} Y_{t j}\right)$ is a GPI for $R$, where the $Y_{i j}$ are distinct indeterminates. By [2], $f\left(\sum_{j=1}^{n(1)} a_{1 j} Y_{1 j}, \ldots, \sum_{j=1}^{n(t)} a_{t j} Y_{t j}\right)$ is also a GPI for $U$. In particular, set $Y_{i j}=u_{i j}$ for all $i, j$. Then $f\left(y_{1}, \ldots, y_{t}\right)=0$ as desired. This proves the lemma.

Lemma 3. Let $R$ be a prime ring and $\rho$ be a nonzero right ideal of $R$. Suppose that $a_{1}, a_{2}, \ldots, a_{t} \in U$ satisfy the following condition: if $\alpha_{1}, \alpha_{2}, \ldots, \alpha_{t} \in C$ satisfy $\left(\alpha_{1} a_{1}+\cdots+\alpha_{t} \alpha_{t}\right) \rho=0$, then $\alpha_{i}=0$ for all $i$. Then there exists an element $u \in \rho$ such that $a_{1} u, \ldots, a_{t} u$ are $C$-independent unless $R$ is a PI-ring.

Proof. Since $R$ is a prime ring, $C$ itself is a field. Define $T_{i} \in \operatorname{Hom}_{C}(\rho C, U)$ by $T_{i}(y)=a_{i} y$ for all $y \in \rho C, 1 \leq i \leq t$. Then $T_{1}, \ldots, T_{t}$ are $C$ independent. Indeed, let $\beta_{1}, \ldots, \beta_{t} \in C$ be such that $\beta_{1} T_{1}+\cdots+\beta_{t} T_{t}=0$. That is, $\left(\beta_{1} a_{1}+\cdots+\beta_{1} a_{t}\right) \rho C=0$. By our assumption, $\beta_{1}=\cdots=\beta_{t}=0$. 
By [3, Lemma 2], either there exists $u \in \rho$ such that $a_{1} u, \ldots, a_{t} u$ are $C$ independent, or there exists $\sum_{i=1}^{t} \delta_{i} T_{i} \neq 0$, where $\delta_{i} \in C$, which is of finite rank. If the first case occurs, then we are done. Therefore we assume the second situation. This implies that $\operatorname{dim}_{C} v \rho C<\infty$ and $v \rho C \neq 0$, where $v=\delta_{1} a_{1}+\cdots+\delta_{t} a_{t}$. By [3, Lemma 1], $\operatorname{dim}_{C} R C<\infty$ and hence $R$ is a $P I$-ring. This completes the proof.

Let $R$ be a prime ring and $S$ be a subring of $U$ containing $R$. It is well known that $S C$ is a closed prime algebra over $C$ [5]. Recall that $f \in$ $S C{ }^{*} C C\left\{X_{1}, X_{2}, \ldots\right\}$ is called nontrivial if $f$ is nonzero. By a result of Martindale [8], if $R$ satisfies a nontrivial GPI with coefficients in $R C$ (in fact, in $U$ ), then $R C$ is a strongly primitive ring. However, a nontrivial GPI for a right ideal $\rho$ of $R$ may only give a trivial identity for $\rho$. For instance, if there exist two $C$-independent elements $a, b \in U$ and $\beta \neq 0$ in $C$ such that $(b+\beta a) \rho=0$, then $a X_{1} b X_{2}+\beta a X_{1} a X_{2}$ is a nontrivial GPI for $\rho$ but gives a trivial identity for $\rho$. Therefore to handle this situation we must give a suitable adaptation for the idea of GPIs satisfied by one-sided ideals. We now follow a notion given by Chuang [3, p. 725]. Let $B$ be a set of $C$-independent elements of $S$. By a $B$-monomial, we mean a monomial of the form $u_{0} Y_{1} u_{1} Y_{2} \cdots Y_{n} u_{n}$, where $\left\{u_{0}, u_{1}, \ldots, u_{n}\right\} \subseteq B$ and where $\left\{Y_{1}, \ldots, Y_{n}\right\} \subseteq\left\{X_{1}, X_{2}, \ldots\right\}$. Thus for each nonzero $f \in S C{ }^{*} C C\left\{X_{1}, X_{2}, \ldots\right\}$ there exists a $B$ such that $f$ is a $C$-linear combination of $B$-monomials. A generalized polynomial $0 \neq f \in$ $S C *_{C} C\left\{X_{1}, X_{2}, \ldots\right\}$ is called a proper $G P I$ for a right ideal $\rho$ of the prime ring $R$ if $f$ is of the form $g\left(X_{1}, \ldots, X_{t}\right) X_{t+1}$, where $g \in S C{ }^{*} C C\left\{X_{1}, X_{2}, \ldots\right\}$, such that $g$ lies in the $C$-span of $B$-monomials for some $B$, a finite set of $C$-independent elements of $S$, and furthermore if $B$ satisfies the following condition:

If $\alpha_{1}, \ldots, \alpha_{l} \in C$ satisfy $\left(\alpha_{1} b_{1}+\cdots+\alpha_{l} b_{l}\right) \rho=0$, where $B=\left\{b_{1}, \ldots, b_{l}\right\}$, then $\alpha_{1}=\cdots=\alpha_{l}=0$.

We remark that two right ideals $\rho_{1}$ and $\rho_{2}$ of the prime ring $R$ satisfy the same GPIs with coefficients in $S C$ if and only if $\rho_{1}$ and $\rho_{2}$ satisfy the same GPIs of the form $g\left(X_{1}, \ldots, X_{t}\right) X_{t+1}$, where $g \in S C{ }^{*_{C}} C\left\{X_{1}, X_{2}, \ldots\right\}$. Indeed, we need only give the proof of the "if" part. Let $f \in S C *_{C} C\left\{X_{1}, X_{2}, \ldots\right\}$ be a GPI for $\rho_{1}$. Let $X_{1}, \ldots, X_{t}$ be all indeterminates occurring in $f$. Fix any element $a \in S$. Then $f\left(X_{1}, \ldots, X_{t}\right) a X_{t+1}$ is a GPI for $\rho_{1}$. By our assumption, $f\left(X_{1}, \ldots, X_{t}\right) a X_{t+1}$ is also a GPI for $\rho_{2}$. Thus $f\left(x_{1}, \ldots, x_{t}\right) S C x_{t+1}=$ 0 for all $x_{1}, \ldots, x_{t+1} \in \rho_{2}$. By the primeness of $S C, f\left(x_{1}, \ldots, x_{t}\right)=0$ for all $x_{1}, \ldots, x_{t} \in \rho_{2}$. That is, $f$ is a GPI for $\rho_{2}$. This proves our remark.

Lemma 4. Let $R$ be a prime ring, $S$ a subring of $U$ containing $R$ and $\rho_{1}, \rho_{2}$ two right ideals of $R$ such that $l_{S}\left(\rho_{1}\right)=l_{S}\left(\rho_{2}\right)$. Suppose that $\rho_{i}$ has no proper GPI in $S C *_{C} C\left\{X_{1}, X_{2}, \ldots\right\}$ for $i=1,2$. Then $\rho_{1}$ and $\rho_{2}$ satisfy the same GPIs with coefficients in $S C$.

Proof. We note first that $l_{S C}\left(\rho_{1}\right)=l_{S C}\left(\rho_{2}\right)$. Indeed, if $y \rho_{1}=0$ where $y \in S C$, then there exists a nonzero ideal $I$ of $R$ such that $I y \subseteq S$, since $R \subseteq S \subseteq U$, and hence $(I y) \rho_{1}=0$, which implies $I y \rho_{2}=0$. Thus $y \rho_{2}=0$ follows. This proves $l_{S C}\left(\rho_{1}\right) \subseteq l_{S C}\left(\rho_{2}\right)$. Thus $l_{S C}\left(\rho_{1}\right)=l_{S C}\left(\rho_{2}\right)$.

Let $0 \neq f \in S C *_{C} C\left\{X_{1}, X_{2}, \ldots\right\}$ be a GPI for $\rho_{1}$. Assume for the moment that $f$ is of the form $h\left(X_{1}, \ldots, X_{l}\right) X_{l+1}$, where $h \in S C *_{C} C\left\{X_{1}, X_{2}, \ldots\right\}$. Then there is a finite set $B$ of $C$-independent elements of $S$ such that $h$ lies 
in the $C$-span of $B$-monomials. Say that $B=\left\{b_{1}, \ldots, b_{t}\right\}$. We proceed by induction on $t$, the number of elements in $B$. Since $\rho_{1}$ has no proper GPI in $S C{ }^{*} C C\left\{X_{1}, X_{2}, \ldots\right\}$, we may assume that $\left(\alpha_{1} b_{1}+\cdots+\alpha_{t-1} b_{t-1}+\alpha_{t} b_{t}\right) \rho_{1}=0$ for some $\alpha_{1}, \ldots, \alpha_{t} \in C$, not all zero. Without loss of generality we can assume $\alpha_{t}=1$. Then we have $\left(\alpha_{1} b_{1}+\cdots+\alpha_{t-1} b_{t-1}+b_{t}\right) \rho_{2}=0$ since $l_{S C}\left(\rho_{1}\right)=l_{S C}\left(\rho_{2}\right)$. Also, let $g$ be the GP obtained from $h$ by replacing the coefficient $b_{t}$ in $f$ by $-\left(\alpha_{1} b_{1}+\cdots+\alpha_{t-1} b_{t-1}\right)$. Set $B_{0}=\left\{b_{1}, \ldots, b_{t-1}\right\}$. Then $g X_{l+1}$ is also a GPI for $\rho_{1}$ and furthermore $g$ lies in the $C$-span of $B_{0}$-monomials, where $\left|B_{0}\right|=t-1$. Applying the induction hypothesis yields that $g X_{l+1}$ is a GPI for $\rho_{2}$. Now the fact that $\left(\alpha_{1} b_{1}+\cdots+\alpha_{t-1} b_{t-1}+b_{t}\right) \rho_{2}=0$ implies that $f$ is a GPI for $\rho_{2}$. Similarly, by the assumption that $\rho_{2}$ has no proper GPI in $S C *_{C}$ $C\left\{X_{1}, X_{2}, \ldots\right\}$ we deduce that every GPI of the form $h\left(X_{1}, \ldots, X_{l}\right) X_{l+1}$ in $S C *_{C} C\left\{X_{1}, X_{2}, \cdots\right\}$ for $\rho_{2}$ is satisfied by $\rho_{1}$. Therefore, $\rho_{1}$ and $\rho_{2}$ satisfy the same GPIs with coefficients in $S C$ by the remark given before this lemma. The proof of Lemma 4 is complete.

With Lemma 4 in hand we are now able to prove the Main Theorem when $R$ is a prime ring.

Lemma 5. The Main Theorem holds when $R$ is a prime ring.

Proof. We may assume that $\rho_{1} \neq 0$ and $\rho_{2} \neq 0$. By Lemma 4, we may assume that $\rho_{1}$ has a proper GPI $f \in S C{ }^{*} C C\left\{X_{1}, X_{2}, \ldots\right\}$. Write $f=$ $g\left(X_{1}, \ldots, X_{l}\right) X_{l+1}$. Thus there exists a finite set $B=\left\{b_{1}, \ldots, b_{t}\right\}$ of $C$ independent elements of $S$ such that $g$ lies in the $C$-span of $B$-monomials. Also, we have that if $\left(\alpha_{1} b_{1}+\cdots+\alpha_{t} b_{t}\right) \rho_{1}=0$ where $\alpha_{i} \in C$, then $\alpha_{1}=\cdots=$ $\alpha_{t}=0$. We claim that $R$ satisfies a nontrivial GPI with coefficients in $U$. If $R$ is a PI-ring, the claim holds trivially. Suppose that $R$ is not a PI-ring. Then by Lemma 3 there exists an element $u \in \rho_{1}$ such that $b_{1} u, \ldots, b_{t} u$ are $C$-independent. Then $g\left(u X_{1}, \ldots, u X_{l}\right) u X_{l+1}$ is a nontrivial GPI for $R$ since $u R \subseteq \rho_{1}$. This proves the claim. By Chuang's theorem [3], $R$ and $S$ satisfy the same GPIs with coefficients in $U$. By Martindale's theorem [8], $S C$ is a strongly primitive ring. In particular, $\operatorname{Soc}(S C)$, the socle of $S C$, is nonzero. Set $\sigma=\operatorname{Soc}(S C) \neq 0$. Then $\sigma$ is a simple ring with minimal right ideals.

Note that $\rho_{1} \sigma$ and $\rho_{1}$ satisfy the same GPIs with coefficients in $U$. Indeed, by Lemma 2, a GPI for $\rho_{1}$ is satisfied by $\rho_{1} \sigma$. Conversely, let $h\left(X_{1}, \ldots, X_{k}\right)$ be a GPI for $\rho_{1} \sigma$ with coefficients in $U$. Fix $k$ elements $y_{1}, \ldots, y_{k} \in \rho_{1}$. Then $h\left(y_{1} X_{1}, \ldots, y_{k} X_{1}\right)$ is a GPI for $\sigma$. Since $\sigma_{R}$ is a dense submodule of $U_{R}$, by [3] $U$ satisfies $h\left(y_{1} X_{1}, \ldots, y_{k} X_{1}\right)$. In particular, set $X_{1}=1$. Then $h\left(y_{1}, \ldots, y_{k}\right)=0$. Therefore $h\left(X_{1}, \ldots, X_{k}\right)$ is a GPI for $\rho_{1}$. This proves that $\rho_{1} \sigma$ and $\rho_{1}$ satisfy the same GPIs with coefficients in $U$. Of course, $\rho_{2} \sigma$ and $\rho_{2}$ also satisfy the same GPIs with coefficients in $U$.

Assume first that $l_{S}\left(\rho_{1}\right)=l_{S}\left(\rho_{2}\right)$, and let $f \in S C *_{C} C\left\{X_{1}, X_{2}, \ldots\right\}$ be a GPI for $\rho_{1}$. Write $f=f\left(X_{1}, \ldots, X_{l}\right)$. Let $t \in \sigma$. Then $t f\left(X_{1} t, \ldots, X_{l} t\right) \in$ $\sigma *_{C} C\left\{X_{1}, X_{2}, \ldots\right\}$ is a GPI for $\rho_{1} \sigma$. Let $d_{1}, \ldots, d_{m}$ be the coefficients occurring in $t f\left(X_{1} t, \ldots, X_{l} t\right)$. Note that $d_{i} \in \sigma$ for each $i$. By Litoff's theorem [7, Theorem 3,p. 90], there exists an idempotent $e \in \sigma$ such that $d_{i} \in$ $e \sigma e$ for $i=1,2, \ldots, m$. Thus $e \rho_{1} \sigma e$ satisfies the GPI $t f\left(X_{1} t, \ldots, X_{l} t\right)$. It follows from the fact $l_{S}\left(\rho_{1}\right)=l_{S}\left(\rho_{2}\right)$ that $l_{\sigma}\left(\rho_{1} \sigma\right)=l_{\sigma}\left(\rho_{2} \sigma\right)$ and hence $l_{e \sigma e}\left(e \rho_{1} \sigma e\right)=l_{\text {eqe }}\left(e \rho_{2} \sigma e\right)$. Note that $e \sigma e$ is now a simple Artinian ring and that $e \rho_{1} \sigma e$ and $e \rho_{2} \sigma e$ are two right ideals of $e \sigma e$. Applying Lemma 1, we 
have $e \rho_{1} \sigma e=e \rho_{2} \sigma e$. Now $e \rho_{2} \sigma e$ satisfies the GPI $t f\left(X_{1} t, \ldots, X_{l} t\right)$ and hence $\rho_{2} \sigma$ satisfies $t f\left(X_{1} t, \ldots, X_{l} t\right)$.

So if we fix $l$ elements $x_{1}, \ldots, x_{l} \in \rho_{2} \sigma$, then $\sigma$ satisfies the GPI $X_{1} f\left(x_{1} X_{1}, \ldots, x_{l} X_{1}\right)$. Since $\sigma_{R}$ is a dense submodule of $U_{R}$, by [3] $U$ satisfy $X_{1} f\left(x_{1} X_{1}, \ldots, x_{l} X_{1}\right)$. In particular, set $X_{1}=1$. Then $f\left(x_{1}, \ldots, x_{l}\right)=0$. Therefore $\rho_{2} \sigma$ and hence $\rho_{2}$ satisfy $f\left(X_{1}, \ldots, X_{l}\right)$. Up to now we have proved that every GPI in $S C{ }^{*} C C\left\{X_{1}, X_{2}, \ldots\right\}$ for $\rho_{1}$ is also a GPI for $\rho_{2}$. Thus $\rho_{1}$ and $\rho_{2}$ satisfy the same GPIs with coefficients in $S C$.

For the converse, let $x \rho_{1}=0$ where $x \in S$. Then $\rho_{1}$ satisfies $x X_{1} \in S C * C$ $C\left\{X_{1}, X_{2}, \ldots\right\}$. By the assumption, $x \rho_{2}=0$. Therefore, $l_{S}\left(\rho_{1}\right) \subseteq l_{S}\left(\rho_{2}\right)$. Similarly, $l_{S}\left(\rho_{2}\right) \subseteq l_{S}\left(\rho_{1}\right)$, and so $l_{S}\left(\rho_{1}\right)=l_{S}\left(\rho_{2}\right)$. This completes the proof.

To prove the Main Theorem we must generalize Lemma 5 to the case of semiprime rings. To arrive at this aim we need some results about orthogonal completions for semiprime rings given in [1]. Let $R$ be a semiprime ring. Recall that a subset $T \subseteq U$ is called orthogonally complete if $0 \in T$ and given any set of orthogonal idempotents $\left\{e_{\omega}\right\} \subseteq C$ and any subset $\left\{x_{\omega}\right\} \subseteq T, \omega \in \Omega$, there exists $x \in T$ such that $e_{\omega} x=e_{\omega} x_{\omega}$ for all $\omega \in \Omega$. For any subset $K \subseteq U$, denote by $\widehat{K}$ the orthogonal completion of $K$ in $U$, which is defined as the intersection of all orthogonally complete subsets of $U$ containing $K$. Note that $\widehat{K}$ itself is an orthogonally complete subset of $U$. Now we prove

Lemma 6. Let $R$ be a semiprime ring, $S$ a subring of $U$ containing $R$ and $\rho$ a right ideal of $R$. Then the following statements hold.

(i) $\rho$ and $\hat{\rho}$ satisfy the same GPIs with coefficients in $U$.

(ii) For any two right ideals $\rho_{1}, \rho_{2}$ of $R, l_{S}\left(\rho_{1}\right)=l_{S}\left(\rho_{2}\right)$ if and only if $l_{\widehat{S}}\left(\hat{\rho}_{1}\right)=l_{\widehat{S}}\left(\hat{\rho}_{2}\right)$.

Proof. For (i), let $f\left(X_{1}, \ldots, X_{l}\right) \in U *_{C} C\left\{X_{1}, X_{2}, \ldots\right\}$ be a GPI for $\rho$. To prove that $f$ is a GPI for $\hat{\rho}$ it suffices to assume that $f$ only involves one indeterminant, say $f=f(X)$. For $x \in \hat{\rho}$, by the definition of $\hat{\rho}$ we have $x=\sum_{\omega}^{\perp} e_{\omega} x_{\omega}$, where $\left\{e_{\omega}\right\}_{\omega \in \Omega}$ is a set of orthogonal idempotents of $C$ such that $\sum_{\omega} C e_{\omega}$ is an essential ideal of $C$ and where $x_{\omega} \in \rho$ for all $\omega \in \Omega$ [1]. Note that $f$ contains no constant term. Thus we have

$$
e_{\omega} f(x)=f\left(e_{\omega} x\right)=f\left(e_{\omega} x_{\omega}\right)=e_{\omega} f\left(x_{\omega}\right)=0
$$

for all $\omega \in \Omega$, since $e_{\omega} x=e_{\omega} x_{\omega}$ and $f\left(x_{\omega}\right)=0$. This implies $f(x)\left(\sum_{\omega} C e_{\omega}\right)$ $=0$. By [1, Lemma 1] $U_{C}$ is a nonsingular $C$-module, which implies $f(x)=0$. This proves (i).

For (ii), assume first that $l_{S}\left(\rho_{1}\right)=l_{S}\left(\rho_{2}\right)$. Let $x=\sum_{\omega}^{\perp} e_{\omega} x_{\omega} \in \widehat{S}$ satisfy $x \hat{\rho}_{1}=0$, where $\sum_{\omega} C e_{\omega}$ is an essential ideal of $C$ and $x_{\omega} \in S$ for all $\omega$. Then $e_{\omega} x \rho_{1}=0$, that is, $e_{\omega} x_{\omega} \rho_{1}=0$. Note that $l_{S C}\left(\rho_{1}\right)=l_{S C}\left(\rho_{2}\right)$, since $l_{S}\left(\rho_{1}\right)=l_{S}\left(\rho_{2}\right)$. We have $e_{\omega} x_{\omega} \rho_{2}=0$. But $r_{U}\left(e_{\omega} x_{\omega}\right)$ is an orthogonally complete subset of $U$, which implies $e_{\omega} x_{\omega} \hat{\rho}_{2}=0$ and hence $x \hat{\rho}_{2}=0$. In other words, $x \in l_{\widehat{S}}\left(\hat{\rho}_{2}\right)$. Therefore, $l_{\widehat{S}}\left(\hat{\rho}_{1}\right) \subseteq l_{\widehat{S}}\left(\hat{\rho}_{2}\right)$. Similarly, $l_{\widehat{S}}\left(\hat{\rho}_{2}\right) \subseteq l_{\widehat{S}}\left(\hat{\rho}_{1}\right)$ and hence $l_{\widehat{S}}\left(\hat{\rho}_{1}\right)=l_{\widehat{S}}\left(\hat{\rho}_{2}\right)$.

Assume next that $l_{\widehat{S}}\left(\hat{\rho}_{1}\right)=l_{\widehat{S}}\left(\hat{\rho}_{2}\right)$. Since the proof that $l_{S}\left(\rho_{1}\right)=l_{S}\left(\rho_{2}\right)$ is trivial, we omit it.

We are now ready to prove the Main Theorem. 
Proof of the Main Theorem. Note that the "if" part is trivial. Therefore it suffices to prove the "only if" part. Suppose that $l_{S}\left(\rho_{1}\right)=l_{S}\left(\rho_{2}\right)$. By Lemma 6, $l_{\widehat{s}}\left(\hat{\rho}_{1}\right)=l_{\widehat{S}}\left(\hat{\rho}_{2}\right)$. Note that $\widehat{R}$ is also a semiprime ring and that $\widehat{S}$ is a subring of $U$ containing $\widehat{R}$. Moreover, $\hat{\rho}_{1}$ and $\hat{\rho}_{2}$ are two right ideals of $\widehat{R}$. Denote by $B$ the complete Boolean algebra of idempotents of $C$ [1]. Fix a maximal ideal $\Delta$ of $B$. Let $\phi$ be the canonical homomorphism from $U$ onto $U / \Delta U$. By [1, Theorem 1], $\phi(\widehat{R})$ is a prime ring with right ideals $\phi\left(\hat{\rho}_{1}\right)$ and $\phi\left(\hat{\rho}_{2}\right)$. Moreover, $\phi(U)=U / \Delta U$ is a right quotient ring of $\phi(\widehat{R})$ and $\phi(\widehat{R}) \subseteq \phi(\widehat{S}) \subseteq \phi(U)$. We claim that $l_{\phi(\widehat{S})}\left(\phi\left(\hat{\rho}_{1}\right)\right)=l_{\phi(\widehat{S})}\left(\phi\left(\hat{\rho}_{2}\right)\right)$. Let $\phi(x) \in l_{\phi(\widehat{S})}\left(\phi\left(\hat{\rho}_{1}\right)\right)$, where $x \in \widehat{S}$. Then $x \hat{\rho}_{1} \subseteq \Delta U$. Now $x \hat{\rho}_{1}$ is an orthogonally complete subset of $U$ since $\hat{\rho}_{1}$ is. By [1, Lemma 2(3)], there is $e \in B-\Delta$ such that ex $\hat{\rho}_{1}=0$. But $e x \in \widehat{S}$ since $B \widehat{S} \subseteq \widehat{S}$. By the fact that $l_{\widehat{S}}\left(\hat{\rho}_{1}\right)=l_{\widehat{S}}\left(\hat{\rho}_{2}\right)$, we have $\operatorname{ex} \hat{\rho}_{2}=0$, and hence $\phi(x) \in l_{\phi(\widehat{S})}\left(\phi\left(\hat{\rho}_{2}\right)\right)$ by [1, Lemma 2(3)] again. This proves our claim.

Let $f \in S C *_{C} C\left\{X_{1}, X_{2}, \ldots\right\}$ be a GPI for $\rho_{1}$. By Lemma $6, f$ is also a GPI for $\hat{\rho}_{1}$. Denote by $f_{\phi}$ the GP obtained from $f$ via replacing each coefficient occurring in $f$ by its image under $\phi$. Then $f_{\phi}$ has coefficients in $\phi(\widehat{S} C)$ and $f_{\phi}$ is a GPI for $\phi\left(\hat{\rho}_{1}\right)$. Since $\phi(\widehat{R})$ is a prime ring and $l_{\phi(\widehat{S})}\left(\phi\left(\hat{\rho}_{1}\right)\right)=l_{\phi(\widehat{S})}\left(\phi\left(\hat{\rho}_{2}\right)\right)$, by Lemma $5 f_{\phi}$ is also a GPI for $\phi\left(\hat{\rho}_{2}\right)$. Write $f=f\left(X_{1}, \ldots, X_{l}\right)$. Then we have $f\left(x_{1}, \ldots, x_{l}\right) \in \Delta U$ for all $x_{i} \in \hat{\rho}_{2}$. But $\bigcap\{\Delta U \mid \Delta$ is a maximal ideal of $B\}=0$; we obtain $f\left(x_{1}, \ldots, x_{l}\right)=0$ for all $x_{i} \in \hat{\rho}_{2}$. That is, $f$ is a GPI for $\hat{\rho}_{2}$ and hence for $\rho_{2}$. This completes the proof of the Main Theorem.

We conclude this paper with two applications of the Main Theorem. Recall that we denote by $Q$ the two-sided Utumi quotient ring of $R$, a semiprime ring.

Theorem 1. Let $R$ be a semiprime ring and $\rho$ a right ideal of $R$ such that $l_{R}(\rho)=0$. Then $\rho$ and $U$ satisfy the same GPIs with coefficients in $Q$.

Proof. We claim that $l_{Q}(\rho Q)=0$. Indeed, let $x \in Q$ be such that $x \rho Q=0$. Then by the semiprimeness of $Q$ we have $x \rho=0$. By the definition of $Q$, there exists a dense left ideal $\lambda$ of $R$ such that $\lambda x \subseteq R$. Thus $(\lambda x) \rho=0$ and hence $\lambda x \subseteq l_{R}(\rho)=0$. This implies $x=0$. So $l_{Q}(\rho Q)=0=l_{Q}(Q)$. By the Main Theorem, $\rho Q$ and $Q$ satisfy the same GPIs with coefficients in $Q(=Q C)$. But $Q_{R}$ is a dense $R$-submodule of $U_{R}$; applying [2, Theorem 2] and Lemma 2 yields that $\rho$ and $U$ satisfy the same GPIs with coefficients in $Q$. This completes the proof.

Theorem 2. Let $R$ be a semiprime ring and $\rho$ a right ideal of $R$. Then, for each positive integer $m, \rho^{m}$ and $\rho$ satisfy the same GPIs with coefficients in $U$.

Proof. By the Main Theorem, it suffices to prove that $l_{U}(\rho)=l_{U}\left(\rho^{m}\right)$. The fact that $l_{U}(\rho) \subseteq l_{U}\left(\rho^{m}\right)$ is clear. For the converse, let $x \in l_{U}\left(\rho^{m}\right)$. Then $x \rho^{m}=0$. That is, $\rho$ satisfies the GPI $x X^{m}$. By Lemma 2, $x(\rho U)^{m}=0$. Now this implies $(x \rho U)^{m}=0$, since $\rho U x \subseteq \rho U$. By the semiprimeness of $U, x \rho U=0$ follows. Therefore $x \rho=0$. This gives $l_{U}\left(\rho^{m}\right)=l_{U}(\rho)$. The proof is now complete. 
Remark. In Theorem 1, we cannot conclude that $\rho$ and $U$ satisfy the same GPIs with coefficients in $U$ even if $R$ is a domain. Indeed, there exists a domain $R$ but $U$ is not a domain. Choose $a \in U-\{0\}$ such that $r_{U}(a) \neq 0$. Set $\rho=R \cap r_{U}(a)$. Then $\rho$ is a nonzero right ideal of $R$ such that $a \rho=0$, but $a U \neq 0$.

\section{REFERENCES}

1. K. I. Beidar, Rings of quotients of semiprime rings, Vestnik Moskov. Univ. Ser. I Mat. Mekh. 1978, no. 5, 36-42; English transl., Moscow Univ. Math. Bull. 33 (1978), no. 5, 29-34.

2. __ Rings with generalized identities. III, Vestnik Moskov. Univ. Ser. I Math. Mekh. 1978, no. 4, 66-73; English transl., Moscow Univ. Math. Bull. 33 (1978), no. 4, 53-58 .

3. C. L. Chuang, GPIs having coefficients in Utumi quotient rings, Proc. Amer. Math. Soc. 103 (1988), 723-728.

4. __ Differential identities with automorphisms and antiautomorphisms. I, J. Algebra 149 (1992), 371-404.

5. T. S. Erickson, W. S. Martindale, and J. Osbern, Prime nonassociative algebras, Pacific J. Math. 60 (1975), 49-63.

6. C. Faith, Lectures on injective modules and quotient rings, Lecture Notes in Math., vol. 49, Springer-Verlag, Berlin and New York, 1967.

7. N. Jacobson, Structure of rings, 2nd ed., Amer. Math. Soc., Providence, RI, 1964.

8. W. S. Martindale III, Prime rings satisfying a generalized polynomial identity, J. Algebra 12 (1969), 576-584.

Department of Mathematics, National Taiwan University, Taipei, Taiwan 10764 